\title{
Design an development of a high performance micro-CT system for small animal imaging
}

\author{
E. Lage, J. J. Vaquero IEEE senior member, S. Redondo, M. Abella, G. Tapias, A. Udías, M. Desco
}

\begin{abstract}
The goal of this work was the development of a lowcost micro-CT scanner, which could be used as an add-on in our previously developed PET systems for small-animals. The scanner design consists of a single-processor computer controlling a micro-focus $\mathrm{X}$-ray tube and a flat panel detector, assembled in a common rotating gantry. The geometrical configuration was selected to achieve a spatial resolution of about $12 \mathrm{lp} / \mathrm{mm}$ with a field of view appropriate for small animals such as mice and rats. The radiated dose is controlled during the acquisition by two different elements: an aluminium filter and a tungsten shutter, attached to the $X$-ray source. The shutter is controlled by the computer in synchronism with the gantry rotation and the detector image integration. In order to achieve high performance with regards to per-animal screening time and cost, the acquisition protocol is able to take advantage from the highest frame rate of the detector also performing onthe-fly corrections for the detector raw data. These corrections include geometrical misalignments, sensor non-uniformities and defective elements, as well as conversion to attenuation images. An FDK reconstruction algorithm adapted to the specific conebeam geometry has been implemented. Symmetries are exploited to accelerate the algorithm and fast back-projection techniques have been developed for those protocols where high resolution is not a requirement.
\end{abstract}

\section{INTRODUCTION}

$T$ HE use of nuclear medicine techniques like PET in small laboratory animals is of increasing relevance in biomedical research. However, these studies are sometimes difficult to interpret due to an ambiguous localization of the tracer uptake. To avoid this problem, registration of PET images with accurate anatomical images, such as X-ray micro-CT has been proven as an appropriate choice in new multimodality systems. For this reason, multimodality imaging systems which are able to carry out combined and intrinsically registered studies was developed during last years for humans. This work reports on a micro-CT scanner based on a solid state semiconductor X-ray sensor and a micro-focus X-ray source, which has been developed to be used as an add-on for our previously developed PET systems for small-animal imaging.

Manuscript received November 17, 2006. This work was supported in part by the Spanish Ministerio de Educación y Ciencia under Grant No. TEC200407052-C02, la Comunidad de Madrid Grant No. GR/SAL/024104 CD Team, and the CENIT program of the Spanish Ministerio de Industria.

Eduardo Lage is with the Unidad de Medicina y Cirugía Experimental, Hospital GU Gregorio Marañon, Madrid, CO 28007, Spain (Corresponding author phone: (+34) 914265 067, e-mail: elage@umce.hggm.es).

Juan José Vaquero, Santiago Redondo, Mónica Abella, Gustavo Tapias, Ángel Udías and Manuel Desco are with the Unidad de Medicina y Cirugía Experimental, Hospital GU Gregorio Marañon, Madrid, Spain.

\section{SYSTEM DESCRIPTION}

The scanner design includes a single-processor computer controlling a micro-focus X-ray tube and a CMOS flat panel detector, assembled in a common rotating gantry. We also included in the prototype a linear motion stage to displace the object or animal being scanned along the field of view and therefore, enabling the tomograph to perform whole body scans (Figure 1).

The system elements are controlled during data acquisition by a single-processor computer (AMD Opteron $2421.6 \mathrm{GHz}$, 1 Gigabyte of RAM, OS Linux 2.4.20) to synchronize the gantry motions with the image integration in the detector. The data acquired for each angular position are captured by means of a digital frame grabber, which serves as an interface with the acquisition software. During the studies, the software protocol is able to process on-line the raw data from the detector, eliminating detector non-uniformities and obtaining a corrected attenuation image for each angular position of the gantry.

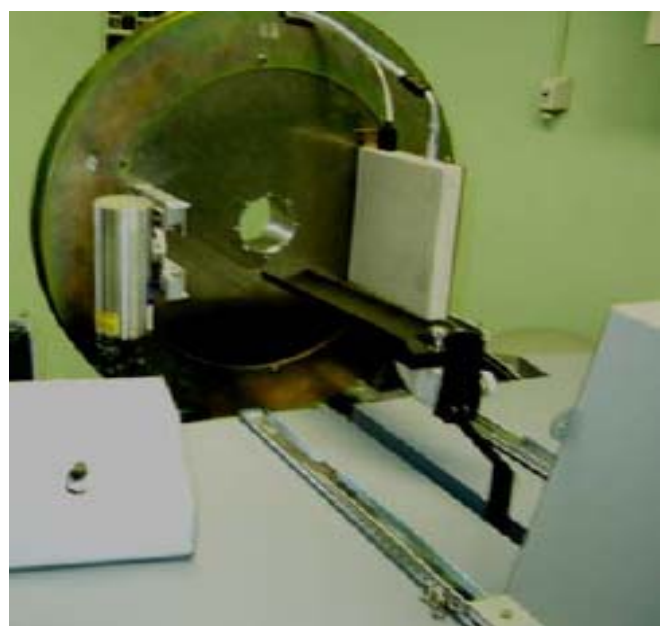

Figure 1. Photograph of the micro-CT prototype showing the different system components. Cone Beam X-ray generated by the X-ray tube (Left) penetrates the object on bed and hits the digital imaging sensor (right). The rotating stage enables the acquisition of complete data sets for the reconstruction algorithms.

\section{A. Source and detector}

The source is an Oxford Instruments (Apogee Family) micro-focus X-ray tube with a stationary tungsten anode and a 35 micrometers focal spot size. The working settings for this component ( 0 up to $50 \mathrm{kV}$ with a maximum power of $50 \mathrm{~W}$ ) are managed from the control computer through a hardware/software interface based on the $\mathrm{I} 2 \mathrm{C}$ bus.

In order to reduce the radiated dose during scans, we added two different parts to the source output window: 
1. Tungsten shutter: Used to interrupt the X-ray beam during the intervals in which the detector is not integrating a valid image, i.e., when the gantry is moving.

2. Aluminium filter: There are a set of interchangeable aluminium plates with different thicknesses (from 0.1 to $1 \mathrm{~mm}$ ) which can be used for different scan protocols. The utility of this element is basically to filter the low energy zone of the emission spectrum in order to obtain a more monochromatic beam.

The detector is placed in a plane orthogonal to the source, thus conforming together a cone beam geometry. The detector covers an active area of $12 \times 12$ centimeters, allowing for pixel sizes of 200, 100 and 50 micrometers depending on the hardware binning selected. This component is connected to the control computer through a frame-grabber card (PIXCI D2X, Epix Inc., Buffalo Grove, IL) which transfers the acquired data to an area of RAM memory accessible for the acquisition software. This configuration makes it possible to achieve transfer rates from 2 up to 8 images per second, depending on the selected binning factor (See Table 1).

TABLE I

IMAGE SENSOR FEATURES (HAMAMATSU C7942)

$\begin{array}{cccc}\text { Pixel size } & \text { Image Size } \\ (\mu \mathrm{m}) & (\text { pixels }) & \begin{array}{c}\text { Data rate } \\ \text { (images } / \mathrm{s})\end{array} & \text { Binning }\end{array}$

\begin{tabular}{cccc}
\hline 50 & $2400 \times 2400$ & 2 & 1 \\
100 & $1200 \times 1200$ & 4 & 2 \\
200 & $600 \times 600$ & 8 & 4 \\
\hline
\end{tabular}

\section{B. System Geometry}

The geometrical configuration was selected in order to accomplish two basic design criteria: system resolution in reconstructed image better than 50 micrometers and a transaxial field of view appropriate for small laboratory rodents (about 7.5 centimeters). The distances from the source to the object $\left(D_{s o}\right)$ and from the object to the detector $\left(D_{o d}\right)$ were selected according to these previously defined criteria, according to the following expressions $[1,2]$ :

$$
\begin{aligned}
& F O V=\frac{\mathrm{T}_{\mathrm{d}}}{\mathrm{M}} \\
& M=\frac{D_{f o}+D_{o d}}{D_{f o}}
\end{aligned}
$$

The final geometry defines a usable field of view of 7.2 centimeters and a magnification factor $(M)$ for the projection images of 1.6 (see figure 2).

The theoretical system resolution is calculated as the convolution of the effects of the finite focal spot size in the source $\left(\rho_{f}\right)$ and the intrinsic resolution of the detector $\left(\rho_{d}\right)$ [2].
The component of the system resolution due to the detector

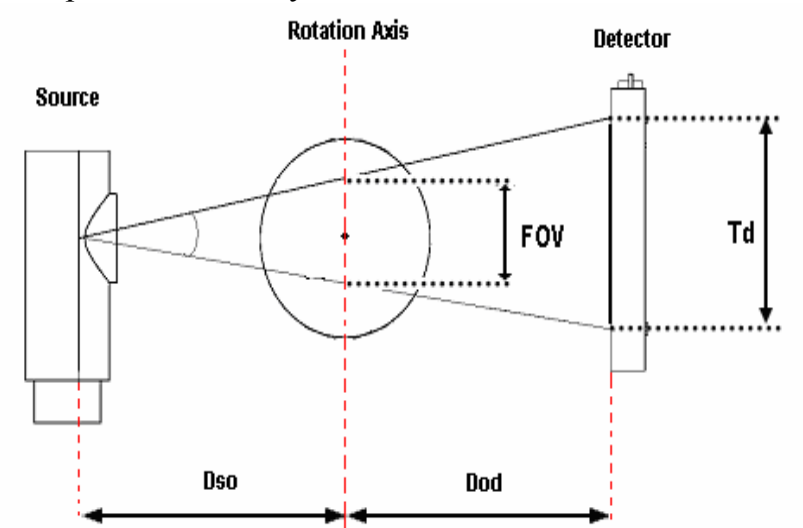

Figure 2. Geometry of the CT system. The chart shows the design parameters used in the calculation of the field of view and the magnification factor. Td refers to the usable size of the detector.

$\left(\theta_{d}\right)$, can be calculated in the center of the field of view using the following expression:

$$
\theta_{d}=\frac{\rho_{d}}{M}
$$

Being $\rho_{f}$ the focal spot size of the source, we can calculate this component $\left(\theta_{f}\right)$ in the center of the field of view as:

$$
\theta_{f}=\rho_{f} \frac{M-1}{M}
$$

The resulting resolution for the reconstructed images can be estimated theoretically supposing Gaussian distributions for $\theta_{f}$ and $\theta_{d}$ according to the formula:

$$
\theta=\sqrt{\theta_{f}^{2}+\theta_{d}^{2}}
$$

For the configuration specified previously we obtain a theoretical spatial resolution of about $12 \mathrm{pl} / \mathrm{mm}$. However, there are additional contributions to the system resolution derived from the tomographic reconstruction process.

The alignment between the source and the detector is a critical factor to achieve the specified resolution value. The fine adjustment of this factor is attained by using an analytical procedure based in the assessment of the elliptical trajectories of two metal spheres, as described in [3]. The correction parameters calculated with this method are applied to the projection images during acquisition time. 


\section{ACQUISITION PROTOCOL}

To take advantage of the maximum detector transfer rate, the acquisition protocol was implemented as an event-driven finite state machine with two possible states. The first one (step) is used to move the gantry to the following angular position and, if needed, to save the data from RAM memory to disk. The second state (shoot) is used to launch the acquisition of either a single frame or a frame sequence in the current angular position and to process the data set acquired in the previous gantry position. The transition between states is triggered in synchronism with the detector integration period $\left(\mathrm{T}_{\mathrm{vc}}\right)$ as is shown in figures 3 and 4 .

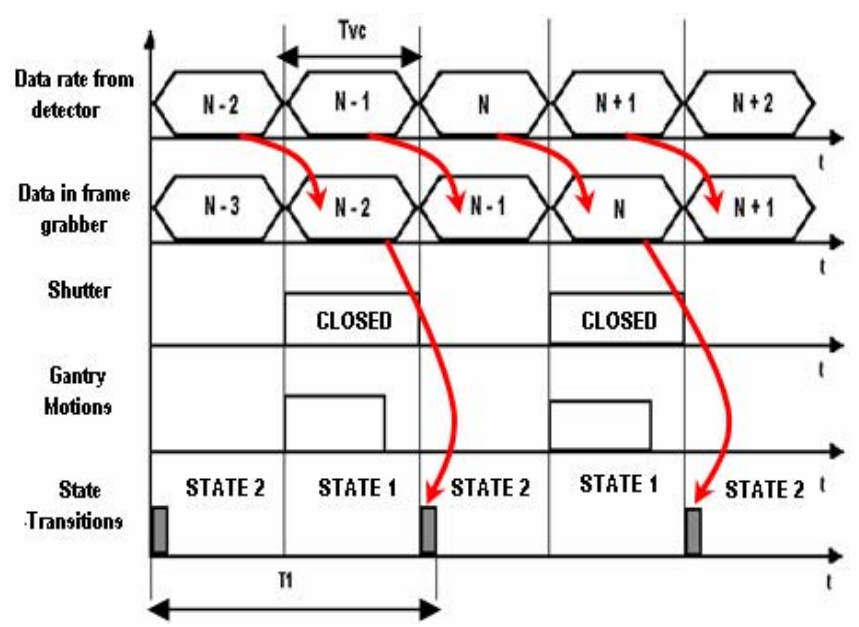

Figure 3. High speed acquisition protocol. Tvc represents the integration period in the detector and T1 indicates the time passed from the acquisition of an image in the detector to the moment in which this image is stored in RAM and is prepared to be saved in the hard disk. The state transitions are shown in the last row of the chronogram. We can also see in this line (in grey), the time spent by the acquisition software during the shoot state, in the processing of the data set acquired in the previous gantry position.

The processing carried out by the software to generate a projection image includes the correction of geometrical misalignments between source and detector, correction of defective lines and pixels of the detector using interpolation with the nearest pixels and the conversion of the resulting data (transmission images with pixel values proportional to the incident photon flux) into attenuation images, as is described in [4]. This latter step includes the dark current correction and its normalization according to a field flood image, previously acquired with the x-ray source settings for the current study and without object between source and detector.

It is also possible to improve the quality of the projection images by acquiring more than one frame in each angular position. In this case the states machine waits in shoot state until the last frame for the current projection arrives. Therefore, and during the wait time, it will be needed an additional processing to average the frames acquired in the previous angular position (Figure 4). Obtaining data with better signal to noise ratio is also achievable by incrementing the number of angular positions acquired during the scan, anyway, it will be necessary a compromised solution between radiated dose and image quality.

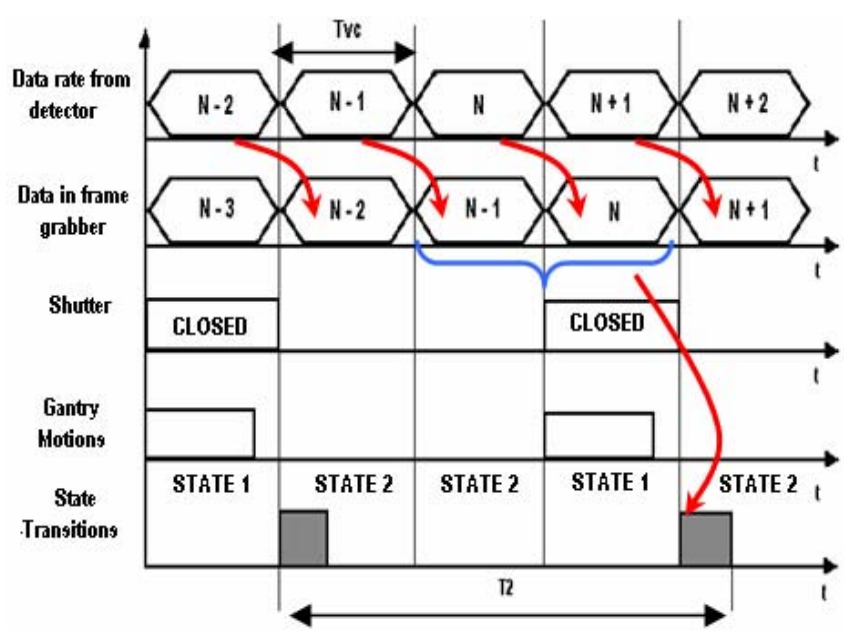

Figure 4. High quality acquisition protocol. In this case the number of acquired frames in each angular position is 2 but is possible to configure this parameter from 2 up to 32 frames per projection. Logically the time spent in data processing during the shoot state (last row in the chronogram) is greater than in the previous case but never greater than Tvc. This last feature allow us to use the following Tve intervals in STATE 2 to carry out additional processing in the projection images such as ramp filtering...

With this implementation, the acquisition time (in seconds) is given by:

$$
T_{a c q}=\left[\frac{1}{F R}\right] \cdot\left[A v_{\text {img }}+N_{\text {loss }}\right] \cdot N_{p} \cdot N_{F O V}
$$

Where:

- $\quad F R$ is the frame rate from the detector, in images per second.

- $A v_{\text {img }}$ is the number of averaged frames per projection image.

- $\quad N_{\text {loss }}$ is the number of frames lost due to the motion of the rotating stage (always 1 in the current implementation).

- $\quad N_{p}$ is the number of views acquired over a 360 degrees gantry rotation span.

- $\quad N_{F O V}$ is the number of axial bed positions

The configuration of these parameters allows for different acquisition protocols: high speed (low resolution and low dose), high resolution (higher dose and acquisition time), etc.

\section{IMAGE RECONSTRUCTION}

To reconstruct the data, we have implemented a Feldkamp algorithm [5] adapted to the specific geometry of our tomograph that includes beam hardening correction, axial shading compensation and calibration in Hounsfield units. Symmetries are exploited to accelerate the algorithm and fast back-projection techniques were developed for those protocols where high resolution is not a requirement. This reconstruction 
software, optimized by the acquisition software preprocessing, leads to reasonable reconstruction times on standard personal computers ( 7 minutes to reconstruct a $512^{3}$ voxels volume in a Pentium $42,80 \mathrm{GHz}$ with $1 \mathrm{~Gb}$ of RAM memory).

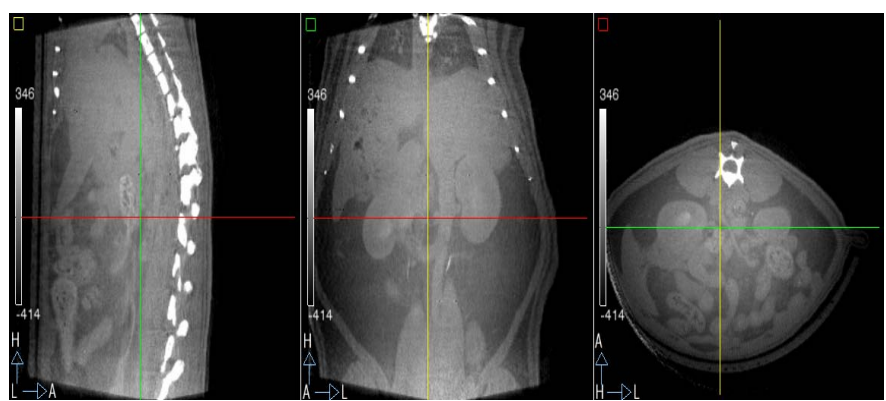

Figure 5. Sagital, coronal and axial views of a mouse abdomen. The main acquisition parameters were $\mathrm{Np}=360$ and $\mathrm{Av}_{\text {imgs }}=8$ with binning 4. Total acquisition time was 6,75 minutes. The image shown has a size of $274 \times 274 \times 288$ pixels. The reconstruction software took 1,5 minutes in an standard personal computer.

\section{CONCLUSIONS}

We have developed a compact micro-CT system, usable as an add-on for our previous PET systems. The use of a CMOS flat panel sensor offers advantages such as large field of view, high spatial resolution and a lower radiated dose.

When using low power X-rays sources, one possible way to improve the SNR ratio and therefore the contrast in soft tissue of the images, is to increase the exposure time. In this context, optimizing the acquisition protocol allow us to obtain a better use of the detector features, improving the system performance with regards to per animal screening time and cost.

\section{ACKNOWLEDGMENTS}

We thank SUINSA Medical Systems for their help in the mechanic of the machines and the integration issues with the PET scanners.

Also we thank Professor Carlos Antoranz from the Physics faculty of the UNED for the facilities offered to access his Xray tomographic system.

\section{REFERENCES}

[1] M. J. Paulus, "High Resolution X-Ray Computed Tomography: An Emerging Tool for Small Animal Cancer Research," Neoplasia, vol. 2, pp. 62-70, 2000.

[2] E. Van de Casteele, "Model-based approach for Beam Hardening Correction and Resolution Measurements in Microtomography"," in Faculty Wetenschappen, Department Natuurkunde Antwerpen: University Antwerpen, 2004.

[3] F. Noo and R. Clackdoyle, "Analytic method based on identification of ellipse parameters for scanner calibration in cone beam tomography," Phys Med Biol., vol. 45, pp. 3489-3508, 2000.

[4] Hamamatsu application Manual, "X-ray flat panel sensor C7912 \& C7942 \& C7943", Mar. 2003, rev 2.10, kr1-i50006.

[5] H. Turbell, "Cone-beam reconstruction using filtered backprojection," Dept. of electrical engineering, pp. Linköping, Universidad de Linköping, 2001. 